\title{
Development of a recombinant Fab-fragment based electrochemical immunosensor for deoxynivalenol detection in food samples
}

\author{
Daniela Romanazzo $^{\mathrm{a}}$, Francesco Ricci ${ }^{\mathrm{a}, \mathrm{f}, *}$, Giulia Volpe ${ }^{\mathrm{a}}$, Christopher T. Elliott $^{\mathrm{b}}$, \\ Silvia Vesco ${ }^{a}$, Katy Kroeger ${ }^{c}$, Danila Moscone ${ }^{a, f}$, Joerg Stroka ${ }^{c}$, Hans Van Egmond ${ }^{d}$, \\ Markus Vehniäinen ${ }^{\mathrm{e}}$, Giuseppe Palleschi ${ }^{\mathrm{a}, \mathrm{f}}$ \\ a Università di Roma, Tor Vergata, Dipartimento di Scienze e Tecnologie Chimiche, Via della Ricerca Scientifica, 00133, Rome, Italy \\ b Institute of Agri-Food and Land Use, School of Biological Sciences, Queen's University Belfast, David Keir Building, Stranmillis Road, Belfast, BT9 5AG, Ireland \\ ${ }^{\mathrm{c}}$ Food Safety E' Quality Unit, Institute for Reference Materials and Measurements, European Commission-Joint Research Centre, Retieseweg 111, B-2440 Geel, Belgium \\ ${ }^{\mathrm{d}}$ Laboratory for Food and Residue Analysis, National Institute for Public Health and the Environment, P.O. Box 1, 3720 BA, Bilthoven, The Netherlands \\ e University of Turku, Department of Biotechnology, Tykistökatu 6A, Biocity 6th floor, FI-20520 Turku, Finland \\ ${ }^{\mathrm{f}}$ Consorzio Interuniversitario Biostrutture e Biosistemi “INBB", Viale Medaglie d'Oro, 305 Roma, Italy
}

\section{A R T I C L E I N F O}

\section{Article history:}

Received 24 February 2010

Received in revised form 19 April 2010

Accepted 20 April 2010

Available online 28 April 2010

\section{Keywords:}

Electrochemical immunosensors

Fab fragment

Toxins

Screen printed

\begin{abstract}
A B S T R A C T
A reliable and cost-effective electrochemical method for the detection of deoxynivalenol (DON) in cereals and cereal-based food samples based on the use of a novel anti-DON Fab fragment is presented. The analytical system employed, Enzyme-Linked-Immunomagnetic-Electrochemical (ELIME) assay, is based on the use of immunomagnetic beads (IMBs) coupled with eight magnetized screen-printed electrodes (8-mScPEs) as electrochemical transducers.

Using standard solutions of DON, a working range between 100 and $4500 \mathrm{ng} / \mathrm{ml}$ was obtained with an $\mathrm{EC}_{50}$ of $380 \mathrm{ng} / \mathrm{ml}$. The ELIME assay was employed to evaluate the cross-reactivity of the Fab fragment towards different trichothecenes revealing a good selectivity towards DON over other trichothecenes with the exception of 3-Ac-DON. The sensor was then applied to cereals and cereal-based food samples (wheat, breakfast cereal and baby-food) and a wide range of sample treatment procedures was tested. Within-laboratory precision (9-24\% repeatability for breakfast cereals and $10-33 \%$ for baby-food) and recovery data ( $82-110 \%$ for breakfast cereals and $97-108 \%$ for baby-food) were calculated by analyzing blank breakfast cereals and baby-foods fortified with DON, demonstrating that the proposed method has the capability for use as a screening assay for DON in such products.
\end{abstract}

(C) 2010 Elsevier B.V. All rights reserved.

\section{Introduction}

Mycotoxins are low-molecular-weight natural products produced as secondary metabolites by filamentous fungi that cause food and feed-borne intoxication and carcinogenic effects in humans and farm animals (Krska et al., 2001). The Fusarium genus is the most frequent fungal contaminant of field corn (Zea mays L.) and represents one of the major causes of mycotoxins production (Lattanzio et al., 2009).

Trichothecenes, a class of mycotoxins produced by Fusarium fungi, are commonly found in cereals and for this reason represent a serious threat for the safety of cereal-based food and feedstuffs. They are classified into Group A and Group B compounds depend-

\footnotetext{
* Corresponding author at: Università di Roma, Tor Vergata, Dipartimento di Scienze e Tecnologie Chimiche, Via della Ricerca Scientifica, 00133, Rome, Italy. Tel.: +39 06 72594403; fax: +3906 72594328.

E-mail address: Francesco.ricci@uniroma2.it (F. Ricci).
}

ing on their structure. The most important type A trichothecenes are T-2 and HT-2, and deoxynivalenol (DON) and nivalenol (NIV) within type $\mathrm{B}$.

Type-B trichothecenes lead to feed refusal, vomiting, anemia, hemorrhage, and immunosuppression (D'Mello et al., 1999) and their concentration in cereals and cereal-based food is severely regulated in the EU (Commission Regulation N. 1126/2007 of 28 September 2007. Off. J. Eur. Union L 255/14).

For DON, the maximum level established in the EU ranges from 200 to $1750 \mu \mathrm{g} / \mathrm{kg}$ depending on the kind of cereal and cereal products. The need to comply with this regulation has increased the interest in the development of robust and reliable instrumental analytical methods and rapid test systems for in-field application. Commonly used methods for trichothecene detection include separating techniques coupled to different detectors (Klötzel et al., 2005; Lattanzio et al., 2009).

During the last decade, much effort has been focused on the development of rapid tests for mycotoxin analysis. Spectrophotometric ELISA kits for DON are for example commercially available 
rapid screening tests to check DON content in grains (Haouet and Altissimi, 2003). These kits present as their main advantages the possibility of processing a large number of samples in a short time period using 'simple to use' instrumentation. The major drawback with these types of procedures is that they can generate both false compliant and false non-compliant results (Anklam et al., 2002).

In an attempt to improve the analytical performances of antibody based techniques, the use of engineered antibody fragment as the recognition element has been proposed as an alternative to whole antibodies. It is well known that antibody engineering techniques such as phage display or enzymatic proteolysis can be used to obtain recombinant Fab fragments, as well as the even smaller Fv (fragment, variable) and single-chain Fv fragments thus leading to better performances of the whole antibodies in respect to affinity, specificity and performance (Valjakka et al., 2002; Näreoja et al., 2009).

Due to their smaller size as functional components of the whole molecule, antibody fragments can offer advantages within immunochemical techniques. The reduction of nonspecific binding resulting from Fc (fragment, crystallizable) interactions and, in solid phase applications, a potentially higher sensitivity in antigen detection as a result of reduced steric hindrance from large protein epitopes are examples. For these reasons, Fab fragments have been recently proposed for several immuoanalytical applications (Dong et al., 2009; Bennett, 2008).

Most of these examples are based on the use of ELISA methods, however electrochemical immunosensors based on the use of Fab fragment as recognition element have also appeared in the literature (Nassef et al., 2009; Vallina-García et al., 2007; Helali et al., 2006). The possible alternative use of electrochemical technique to develop sensitive immunosensors with similar or even better performances of the more classic spectrophotometric ELISA assay has been demonstrated by several groups (reviewed in Ricci et al., 2007).

Advantages of electrochemical measurements over that of spectrophotometric include the possibility of increased speed, miniaturization and multiplexing (Li et al., 2008). The low cost of instrumentation, the possibility of in situ analysis and the insensitivity to turbid samples are among the other advantages of this technique. The use of screen-printed electrodes (ScPEs), which allows the mass production of electrodes at a very low cost is another significant development. More recently, electrochemical sensors have been also successfully coupled with immunomagnetic beads (IMBs) in a device called magnetoelectrochemical immunosensor (Gehring et al., 1999; Piermarini et al., 2009). Using this approach, combining the selectivity of the antibodies, the sensitivity of the electrochemical detection and the possibility of concentrating magnetic particles on the electrode surface, it is possible to achieve remarkable enhancement in the performance of classical immunoassays (Delibato et al., 2005).

All these attributes make the platform a potentially suitable approach to be applied as a screening method with capacity of high sample throughput to screen large numbers of samples.

Here we present the development of an Enzyme-LinkedImmunomagnetic-Electrochemical assay (ELIME) for DON detection in food samples based on the use of magnetic beads and ScPEs. As the recognition element, a recently prepared Fab fragment specific for DON was employed. A wide range of different cereal-based matrices were subjected to a large number of sample treatment procedure. For each matrix, the best sample treatment procedure was selected and recovery and precision data for the method were calculated by analyzing fortified blank samples. A number of naturally contaminated samples were also employed in the validation procedure.

\section{Materials and methods}

\subsection{Materials}

\subsubsection{Reagents}

Biotinylated anti-DON Fab fragment $(1 \mathrm{mg} / \mathrm{ml}$ in $50 \mathrm{mM}$ Tris buffer $+150 \mathrm{mM} \mathrm{NaCl}$, pH 7.8) was obtained from Turku University and produced as described by Korpimäki et al. (2004) Briefly, the antibody genes from an anti-DON monoclonal cell line were cloned as a Fab fragment to bacterial expression vector and during cloning an unpaired cysteine (for biotinylation) and hexahistidine peptide (for purification) were added to the Fab fragment. A high cell density fermentation of RV308 E. coli strain harboring the expression vector was used for production of Fab fragment and the Fab fragment was purified and site-specifically biotinylated with cation exchange chromatography followed by metal affinity chromatography. The monoclonal cell line and DON conjugate (DON-DSC-cHSA) prepared by conjugation with N,N2 disuccinimdyl carbonated (DSC) and carbamylated human serum albumin (cHSA) $(1 \mathrm{mg} / \mathrm{ml})$ were prepared at Queens University, Belfast.

For labeling purpose avidin and biotinylated peroxidase enzymes were used. The solution ( $A B C$ reagent) was purchased as a kit from Pierce (Rockford, IL, USA) (Ultra-Sensitive ABC Peroxidase Staining Kit) and is a mixture of specially purified Avidin and Biotinylated Horse Radish Peroxidase (HRP).

Non-fat dry milk, blotting grade, was from Bio-Rad Laboratories (Hercules, CA, USA). 3,3',5,5'-tetramethybenzidine (TMB) liquid substrate solution (super sensitive form for ELISA), Tween 20 and all other reagents of analytical-reagent grade were obtained from Sigma (St. Louis, MO, USA).

During the optimization of the extraction procedure, Mycosep MultiSep ${ }^{\circledR} 225$ Trich or MultiSep ${ }^{\circledR} 227$ Trich+ and Bond Elut Mycotoxin columns were used to purify the extracted samples. Columns were used following manufacturer instructions.

Tosylactivated Dynabeads ${ }^{\circledR} \mathrm{M}-280\left(2 \times 10^{9}\right.$ beads/ml, average size $2.8 \mu \mathrm{m}$, about $30 \mathrm{mg} / \mathrm{ml}$ ) were from Dynal, Lake Success, NY. Ultra-pure water was produced with a Milli-Q system (Millipore, Bedford, MA, USA).

The following buffer solutions were employed during the ELIME assay. TBS $1 \times(50 \mathrm{mM}$ Tris buffer $+150 \mathrm{mM} \mathrm{NaCl}, \mathrm{pH} 7.8)$ TBS $2 \times(100 \mathrm{mM}$ Tris buffer $+300 \mathrm{mM} \mathrm{NaCl}, \mathrm{pH} 7.8)$ and CPB $(50 \mathrm{mM}$ citrate-phosphate buffer, $\mathrm{pH}$ 5.0).

\subsubsection{Electrodes}

Screen-printed electrodes were produced with a 245 DEK (Weymouth, England) screen-printing machine. Graphite-based ink (Elettrodag 421) from Acheson (Milan, Italy) was used to print the working and counter electrode (Ricci et al., 2006). The substrate was a flexible polyester film (Autostat HT5) obtained from Autotype Italia (Milan, Italy). The diameter of the working electrode was $0.3 \mathrm{~cm}$ resulting in an apparent geometric area of $0.07 \mathrm{~cm}^{2}$. A silver ink was used to print the reference electrode. A strip of eight screen-printed electrodes was connected to the potentiostat by an 8-channel multiplexer. Before use, ScPEs were pre-treated in $0.01 \mathrm{M}$ PBS buffer $\mathrm{pH} 7.4$ by applying an anodic potential of $1.7 \mathrm{~V}$ for $180 \mathrm{~s}$ (a procedure which improves the sensitivity and reproducibility of these electrodes (Ricci et al., 2003)) and kept dried in the dark until ready to use (Ricci et al., 2003).

\subsection{Apparatus}

A rotation device (Dynal sample mixer) and a magnetic particle concentrator (Dynal MPC) were used for the manipulation of the IMBs. 
Neodynium magnets ( $3 \mathrm{~mm} \times 6 \mathrm{~mm}$ ), used to localize the IMBs onto the surface of the ScPE working electrode, were from Italfit Magneti (Fagagna-Udine, Italy).

Amperometric measurements were performed using an Autolab electrochemical system (Eco Chemie, Utrecht, The Netherlands) equipped with PGSTAT-12 and GPES software (Eco Chemie).

Further laboratory equipments include a High speed blender, a Reacti-Therm III Heating module equipped with a Reacti-Vap III evaporator (for sample evaporation under nitrogen stream) purchased from Pierce Biotechnology (Rockford, USA).

\subsection{Procedures}

\subsubsection{Enzyme-Linked-Immunomagnetic-Electrochemical (ELIME) assay}

The detection of DON using the ELIME method is based on the use of magnetic beads as support for the immunological chain. The entire ELIME procedure involves three major steps.

The first step involved magnetic beads being washed and coated with DON conjugate (DON-DSC-cHSA) and stored at $4{ }^{\circ} \mathrm{C}$ when not in use. In the second step, the coated particles are employed for the competitive assay and, when the immunological chain is completed, the particles are pipetted on the surface of eight magnetized screen-printed electrodes for the electrochemical measurement (third step). A detailed description of these steps is summarized in the supporting info section (SI1).

\subsubsection{Evaluation of the matrix effect using different sample treatment procedures}

For the evaluation of the matrix effect on the immunoelectrochemical measurements, several treatment procedures of DON free (blank) samples were performed using types of cereals or cereal products in order to select the best treatment for each matrix. In all cases, after treatment of these blank samples, the extracts were fortified with known amounts of DON in order to construct calibration curves that were compared with the standard curve, produced using the same standard solutions of DON prepared in distilled water. The details of the sample treatment procedures are provided in the supporting info section (SI1) and in Scheme SI1.

\subsubsection{Analysis of fortified and naturally contaminated samples}

The sample treatment procedure based on the use of acetonitrile/water without any clean-up step (Scheme SI1), was selected and employed to analyze fortified and naturally contaminated breakfast cereal and baby-food samples. Dried extracts were reconstituted in $2 \mathrm{ml}$ and $400 \mu \mathrm{l}$ of distilled water for breakfast cereal and baby-food, respectively (see Section SI3 for details).

\section{Results and discussion}

An ELIME assay was developed, based on the use of a recombinant Fab fragment as the recognition element for DON detection in cereal and cereal products such as baby-food and breakfast cereals. This novel Fab fragment was site-specifically biotinylated from single engineered cysteine leading to a highly active label antibody. The ELIME was based on the coupling of a strip of 8-mScPEs with IMBs employed in a competitive indirect assay. In this approach the magnetic beads were coated with the conjugated toxin and a competitive step was performed by adding the free toxin together with the Fab fragment. The Fab fragment, conjugated with a biotin group, was linked to HRP via an avidin-biotin complex system (Scheme 1).

The amount of HRP present was measured by adding $\mathrm{TMB}_{\text {red }}$ and $\mathrm{H}_{2} \mathrm{O}_{2}$. The enzymatic product $\left(\mathrm{TMB}_{\mathrm{Ox}}\right)$ was then electrochemically quantified by using chronoamperometry technique and an 8-mScPEs strip (Scheme 1). It is important to emphasize that all the steps of the immunological chain were performed in solution due to the use of the paramagnetic beads. At the end of the immunological chain, the measurement was carried out on the surface of the electrodes. The 8-mScPEs strip was designed to have a magnet at the bottom of each working electrode; enabling the concentration of the magnetic particles on the surface of the working electrodes to achieve a better sensitivity. Moreover, the use of this innovative 8-ScPEs strip was extremely useful as a prototype device moving towards a fast and automated measurement platform.

The fact that the immunological chain is separated from the electrode surface is not only particularly advantageous to avoid any possible contamination which could poison the electrode surface posing problems for the future electrochemical measurement, but it is also of crucial importance for possible multiplexing applications. The 8-mScPEs strip is in fact adaptable to the detection of other analytes provided that the immunological chain is performed separately.

The indirect competitive immunoassay employed required different experimental steps. The magnetic beads were first coated with the conjugated toxin. Two different conjugated toxins were evaluated. With the first conjugate (i.e. DON-keyhole limpet hemocyanin (KLH)), no electrochemical signals were obtained at the end of the immunological chain. This was probably due to the fact that the toxin conjugation with KLH hampers the antibody/antigen binding preventing the formation of the complex and so the linkage of the HRP signaling label. Another conjugate (DON-DSC-cHSA) was then tested showing improved results and measurable signals at the end of the immunological chain. This conjugate was used in all subsequent experiments.

In order to optimise the competitive assay, different dilutions of specific antibody and conjugate were tested. The binding

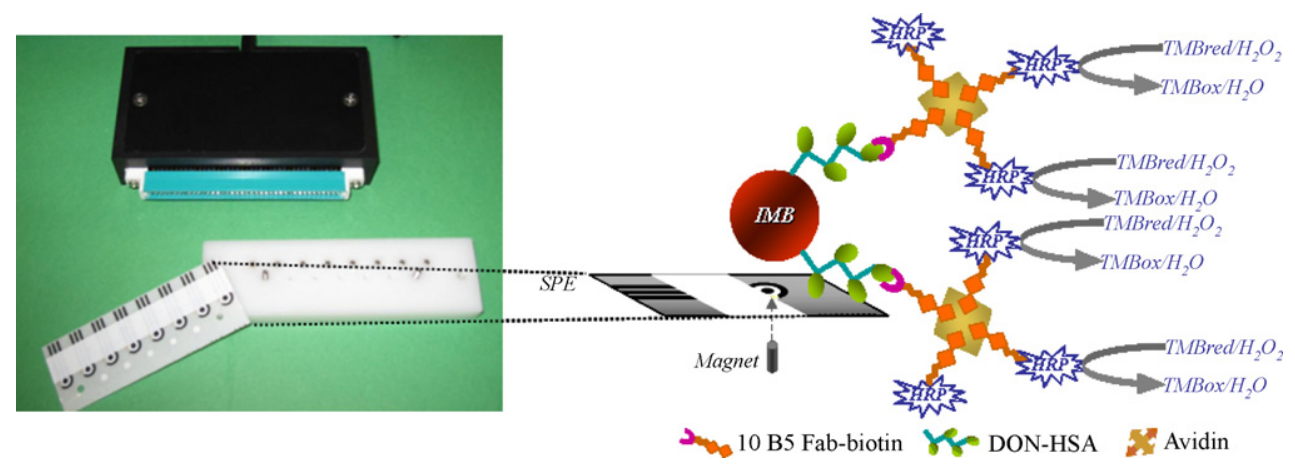

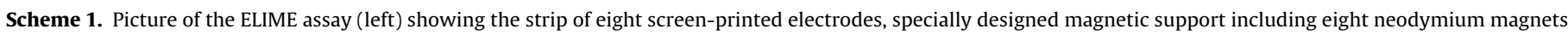

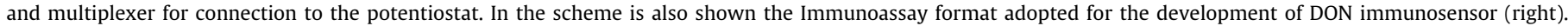

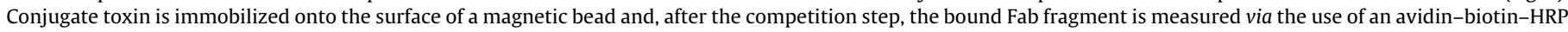

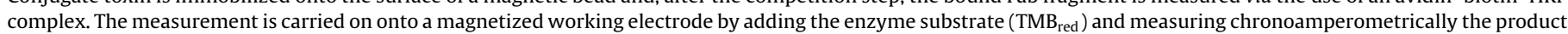
$\left(\mathrm{TMB}_{\mathrm{ox}}\right)$ after $1 \mathrm{~min}$ of incubation. 


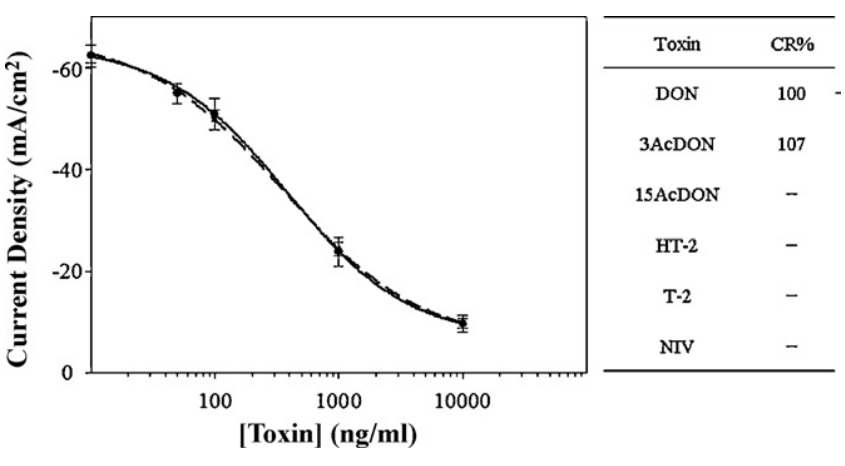

Fig. 1. Standard competition curves obtained with DON (black line) and 3-Ac-DON (dotted line) which shows a strong cross-reactivity of the Fab fragment for 3-AcDON. The embedded table shows the other values of cross-reactivity obtained with other similar toxins demonstrating that the Fab fragment is completely insensitive to NIV toxin and other trichothecenes. Each point is the mean of three measurements.

curve obtained with different Fab dilutions and the selected DONDSC-cHSA concentration $\left(375 \mu \mathrm{g} \mathrm{ml}^{-1}\right)$, in the presence of a fixed amount of avidin-biotin-HRP complex (suggested by product instructions), shows an inflection point at a Fab dilution of 1:7000 $(\mathrm{v}: \mathrm{v})$, which was then selected for the further competitive assay (Fig. SI1).

A standard competition curve was obtained using a range of standard toxin concentrations (Fig. 1). All experimental data were fitted using a "non-linear four-parameter logistic calibration plot" as in the equation:

$f(x)=\frac{a-d}{1+(x / c)^{b}}+d$

in which $a$ and $d$ are the asymptotic maximum and minimum values, $c$ is the value of $x$ at the inflection point and $b$ is the slope.

The detection limit, defined as the concentration corresponding to the $f(x)$ value obtained by subtracting three standard deviations of zero point from the mean of the zero standard measurement (mean value-3SD), was determined to be $63 \mathrm{ng} / \mathrm{ml}$, while the value for the sensitivity, calculated as the amount of DON needed to produce a $50 \%$ decrease in the signal $\left(E_{50}\right)$ was $380 \mathrm{ng} / \mathrm{ml}$. The working range, calculated as the toxin concentration that gives inhibition values between $20 \%$ and $80 \%$, was found to be $100-4500 \mathrm{ng} / \mathrm{ml}$.

The sensitivity of the developed immunosensor was not as high as other previously reported immunosensors targeting different analytes (Piermarini et al., 2007; Ricci et al., 2007). This, however, cannot be ascribed to the fact that a Fab fragment is used instead of a natural antibody. Previous reports with rapid fluorescent polarization immunoassay and conventional ELISA, utilizing a naturally produced anti-DON antibody, showed in fact similar sensitivity (Maragos and Plattner, 2002; Maragos and McCormick, 2000). Of greater importance is that the reported sensitivity in the present method is still suitable for the desired application. The regulatory levels set by the European Commission for DON trichothecenes range from $1750 \mu \mathrm{g} / \mathrm{kg}$ for unprocessed cereal to $200 \mu \mathrm{g} / \mathrm{kg}$ for baby-food (Commission Regulation N. 1126/2007 of 28 September 2007. Off. J. Eur. Union L 255/14).

After optimization of the immuno-electrochemical parameters, the selectivity of the developed immunosensor was evaluated by testing different toxins (Fig. 1). While the response to type-A toxins is negligible as well as that for 15-Ac-DON and NIV, a high cross-reactivity is observed with 3-Ac-DON. This cross-reactivity calculated as $100 x / y$, were $x$ is the amount of DON and $y$ is the amount of 3-Ac-DON required to produce a signal decrease of $50 \%$, was shown to be $107 \%$. This indicated that 3 -Ac-DON could be a strong interference for the accurate measurement of DON. Such cross-reactivities against 3-Ac-DON are common drawback of other antibody based detection methods for DON (Maragos and McCormick, 2000; Schneider et al., 2004; Kolosova et al., 2007). The low sensitivity of the assay for 15 -Ac-DON relative to 3-Ac-DON indicates that modification of DON at the $\mathrm{C}-15$ position inhibited binding, while modification at the $\mathrm{C}-3$ position did not. This could be ascribed to the fact that DON conjugated at the $\mathrm{C}-3$ position was used to obtain the Fab fragment employed in this work. It is important to note that there is no report of anti-DON antibodies which are completely insensitive to $3-\mathrm{Ac}-\mathrm{DON}$ or $15-\mathrm{Ac}-\mathrm{DON}$. This is due to the fact that conjugation to the carrier proteins for antibody recognition has always been to one of these functional groups (Nicol et al., 1993; Sinha and Savard, 1996; Maragos and Plattner, 2002).

No regulatory limit has yet been established for 3-Ac-DON and this can cause some problems concerning false positive (noncompliant) results form the application of this immunosensor in samples containing 3-Ac-DON.

Although the performances of the developed electrochemical immunosensor for DON detection is not improved in respect to what is reported with ELISA or SPR approaches used for DON detection (Tüdös et al., 2003; Schnerr et al., 2002) it has to be stressed the fact that electrochemistry presents several advantages over these methods including the possibility of low-cost instrumentation, miniaturization and multiplexing. Moreover, despite the interest in these analytes demonstrated by the large number of papers published in recent years, few are the reports which deal with complicate food samples such as baby-food or breakfast cereal which not only present a more complex matrix but also lower legislation levels. For these reasons, the performance of the electrochemical immunosensor was evaluated with different cereals and cereal-based foods. The best sample treatment procedure for each food matrix was selected taking in consideration both the regulatory level of the toxin established for each matrix and the effect of possible interferences. After the evaluation of the best sample treatment procedure, the immunosensor was tested with fortified blank materials to evaluate both recovery and precision of the method.

\subsection{Application of the ELIME assay in cereal products}

A rigorous study was performed to evaluate the best sample treatment procedure to be applied to different cereal and cereal-based food samples. For clarity, the table of the European Commission Regulation 1126/2007 for the maximum admissible levels of DON in food products is provided in the supporting info (Table SI1). It is worthy of note that most of the published reports about immunochemical methods for DON detection are applied only to unprocessed cereals such as wheat, oats and maize. Although this is an extremely important application, it is also crucial to demonstrate the applicability of a novel method in more complex matrices where more difficulties will be faced due to the presence of possible interferences and to the lower sensitivity required. For this reason, in the present study three different matrices were selected from those having the highest maximum level of DON (wheat samples) to those with the most stringent regulations (baby-food) to demonstrate the applicability of the proposed method to any cereal-based matrices.

\subsection{Wheat sample}

Wheat samples were tested to evaluate the possible matrix effect on the immunosensor response. While different sample treatments were considered, as the regulatory limit for DON is high $(1750 \mu \mathrm{g} / \mathrm{kg})$, a simple procedure involving the use of pure water as extraction solvent, which has been previously reported to be extremely efficient for polar trichothecenes such as DON and NIV, was assessed (Bretz et al., 2006; Cahill et al., 1999). For the evaluation of the matrix effects, a water extract obtained by 

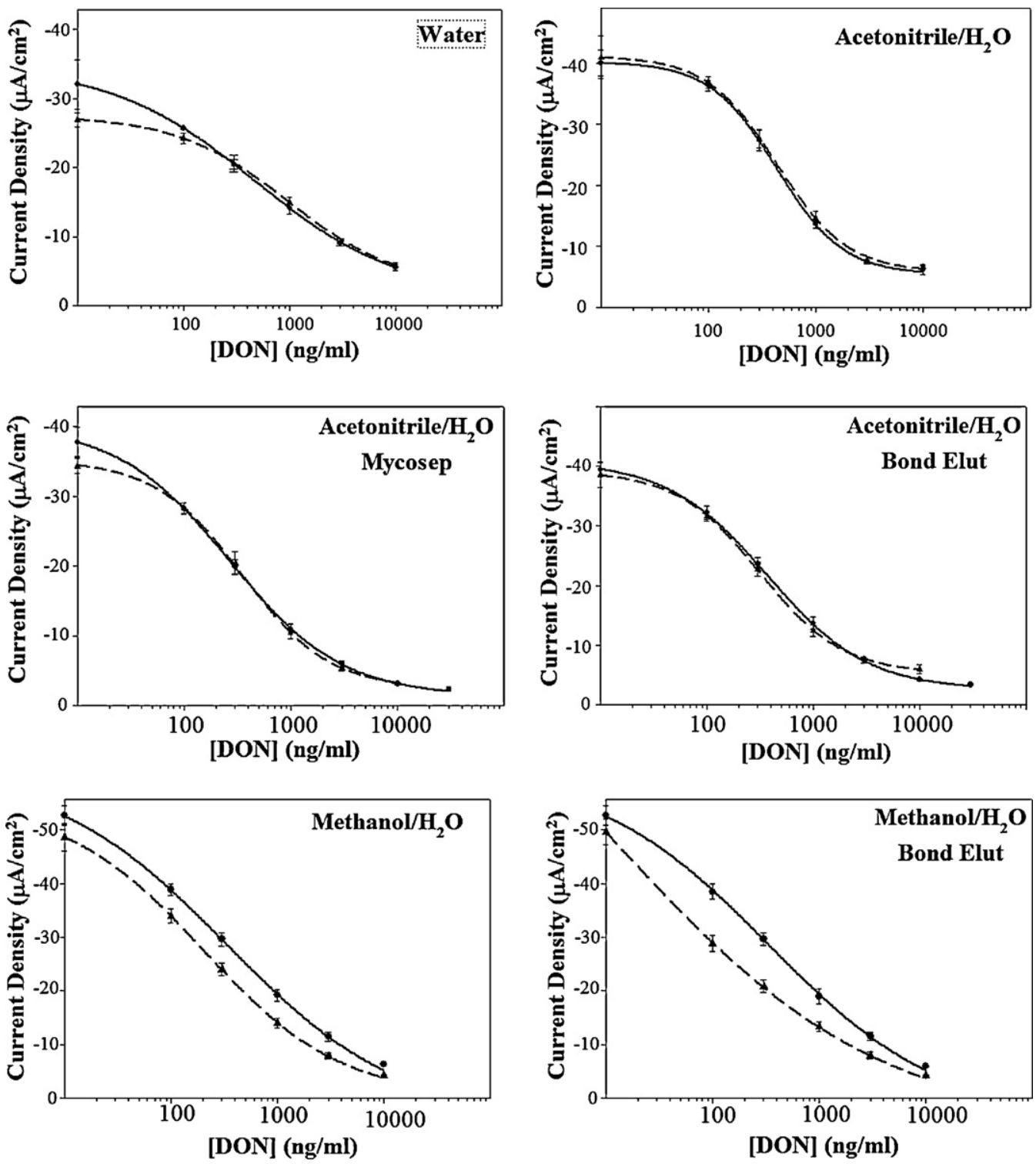

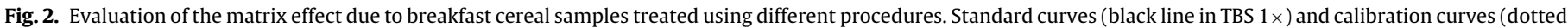
line in blank breakfast cereal extracts) for DON. Each point is the mean of three measurements. See Scheme SI1 for details about sample treatment procedures.

blank wheat samples was spiked with different concentrations of DON and the calibration curve was compared with the standard curve obtained in pure buffer solution (Fig. SI2). A negligible matrix effect was observed, thus demonstrating that the extraction with water can effectively be used for DON detection in unprocessed wheat.

\subsection{Breakfast cereal}

The water-based extraction as applied to wheat was first evaluated. However, in this case, the sensitivity required was higher $(500 \mu \mathrm{g} / \mathrm{kg})$. This value corresponded to the minimum quantifiable concentration of DON by the developed immunosensor, so concentrations slightly below the regulatory limit will not be determined. For this reason, $4 \mathrm{ml}$ of water-based extract were lyophilized and resuspended with only $2 \mathrm{ml}$ of water thus concentrating the extract and giving the possibility of quantify at 0.5 times the regulatory level. This procedure did not affect the immunosensor response and a negligible matrix effect was observed (Fig. 2).

This result was considered important and demonstrates the feasibility of the developed immunosensor for food analysis. However, the lyophilization step is more time consuming and laborious than that desired for a rapid screening test. Therefore, a range of extraction procedure based on the use of organic solvents was tested. It was hoped this would facilitate the delivery of a rapid and clean extract. Five different sample treatment procedures (Scheme SI1) were investigated. Extracting with a solution of acetonitrile/water was studied and the possible utilization of a clean-up step, using Solid Phase Extraction (Mycosep or Bondelut cartridges) was considered (Bretz et al., 2006; Milanez and Valente-Soares, 2006). Methanol was also used as an extraction solvent followed by a Bond Elut clean-up procedure. For all these sample treatment procedures a drying step is required both to concentrate the extract and to avoid the presence of any organic solvent, which would adversely affect the antigen-antibody interaction, and also could pose some problem to the electrochemical measurement.

The immunosensor showed comparable results for all the procedures involving the use of acetonitrile/water (Fig. 2, Scheme SI1). Methanol/water as extraction solvent did not produce promising results and substantial overestimation values were observed (Fig. 2, Scheme SI1). This result is in agreement with a previous report, which demonstrated that acetonitrile/water extraction led 
Table 1

Recovery values of DON immunosensor obtained analyzing fortified blank breakfast cereal samples. The extraction procedure adopted involves the use of acetonitrile/water without any purification step.

\begin{tabular}{clcl}
\hline DON added $(\mu \mathrm{g} / \mathrm{kg})$ & DON found $\pm \mathrm{SD}(\mu \mathrm{g} / \mathrm{kg})$ & $\mathrm{RSD}_{\mathrm{r}} \%$ & Recovery \% \\
\hline 100 & n.d. & - & - \\
300 & $268 \pm 53$ & 20 & 89 \\
500 & $410 \pm 53$ & 12 & 82 \\
750 & $716 \pm 172$ & 24 & 95 \\
1000 & $1098 \pm 98$ & 9 & 110 \\
\hline
\end{tabular}

Table 2

Recovery values of DON immunosensor obtained analyzing experimentally contaminated blank baby-food samples. The extraction procedure adopted involves the use of acetonitrile/water without any purification step.

\begin{tabular}{ccll}
\hline DON added $(\mu \mathrm{g} / \mathrm{kg})$ & DON found $\pm \mathrm{SD}(\mu \mathrm{g} / \mathrm{kg})$ & $\mathrm{RSD}_{\mathrm{r}} \%$ & Recovery \% \\
\hline 100 & $97 \pm 32$ & 33 & 97 \\
200 & $198 \pm 20$ & 10 & 99 \\
400 & $434 \pm 52$ & 12 & 108 \\
1000 & $1050 \pm 157$ & 15 & 105 \\
\hline
\end{tabular}

to a lower co-extraction of interfering matrix compounds than methanol/water solvent (Klötzel et al., 2005).

The applicability of this immunosensor in breakfast cereal, already demonstrated with water extraction, was also confirmed with acetonitrile/water extraction; moreover, the use of a clean-up step did not lead to any significant improvement in respect of what obtained with unpurified extracts. Acetonitrile/water solution was determined to be the best extraction solvent for breakfast cereals without the need of any clean-up step.

\subsection{Baby-food}

In the case of baby-food, the regulatory level set by the European Commission $(200 \mu \mathrm{g} / \mathrm{kg})$ is more stringent than breakfast cereal. Taking in consideration the results previously obtained with breakfast cereals it was determined to test the possible use of acetonitrile/water extraction without the need for any purification step. However, in this case the sensitivity required was even higher than that of breakfast cereal and a concentration step was needed (see "section evaluation of matrix effect using different sample treatment procedures" in supporting info). It is noteworthy that the matrix effect found with the baby-foods extracts was negligible, demonstrating the wide applicability of the immunosensor to different cereal-based food matrices.

\subsection{Recovery test of DON immunosensor with experimentally and naturally contaminated samples}

Experimentally contaminated blank breakfast cereal and babyfood samples were tested to evaluate the recovery and precision of the developed sensors.

Results obtained with both spiked breakfast cereal and babyfood (Tables 1 and 2) confirmed the effectiveness of the developed immunosensor for DON detection with recoveries values ranging from 82 to $110 \%$ for breakfast cereals and from 97 to $108 \%$ for babyfood samples.
An average reproducibility of ca. $16 \%$ was observed with both samples which, being similar to what usually observed with ELISA or electrochemical immunosensor approaches, demonstrate the suitability of the proposed platform as a screening method for DON

Due to the availability of only one naturally contaminated breakfast cereal sample (with known amount of DON determined by HPLC: $485 \pm 61 \mu \mathrm{g} / \mathrm{kg}$ ), it was decided to blend the contaminated sample with increasing amounts of blank sample in order to obtain different concentration levels of DON. These samples were analyzed with the ELIME-strip utilizing the optimized conditions and the experimental results have been reported in Table 3. As shown, recovery values ranging from 89 to $119 \%$ were obtained and these data demonstrated the applicability of the proposed sensors for DON detection in real samples.

\section{Conclusions}

In the present study an ELIME assay based on the use of IMBs coupled with a magnetized 8-ScPEs strip for the detection of DON has been presented. The recognition of DON is made possible by the use of a recently prepared Fab fragment which presents favorable characteristics of sensitivity to DON and a cross-reactivity to 3-Ac-DON comparable to that of natural antibodies (Maragos and McCormick, 2000). The performance of the developed electrochemical system was rigorously evaluated by testing different cereal and cereal-based food samples. Three different matrices were selected to encompass both high (wheat) and low (baby-food) regulatory levels. The best performing sample treatment procedure for each food matrix was selected taking in consideration both the regulatory level of the toxin established for each matrix and the adverse effects of possible interferences. Results obtained during the evaluation of the matrix effect clearly showed that the developed electrochemical immunosensor for DON detection is extremely robust and insensitive to sample matrix interference (wheat, breakfast cereal and baby-food). Although other techniques such as SPR (Tüdös et al., 2003; Schnerr et al., 2002), fluorescent array biosensor (Ngundi et al., 2006) and electronic olfactory system (Olsson et al., 2002) were proposed for the detection of DON in food samples, to our knowledge none of them was challenged with complex matrices such as baby-food and breakfast cereals. We demonstrated here that the detection of DON with the developed immunosensor was possible in all these matrices. A simple water extraction was employed for wheat, while an acetonitrile-water extraction without clean-up coupled with a drying step was selected for breakfast cereal and baby-food.

Precision and recovery of the ELIME-strip was then calculated by analyzing more replicates of blank breakfast cereal and baby-food experimentally contaminated with different amount of DON.

The ELIME-strip was employed to analyze one naturally contaminated breakfast cereal sample blended with increasing amounts of blank sample in order to obtain different concentration levels of DON. All data obtained indicate that the method gave satisfactory results for the determination of DON in breakfast cereal and baby-food. The electrode strip platform employed can be used to perform eight measurements simultaneously which will allow the processing of a high number of samples in a relatively fast time.

Table 3

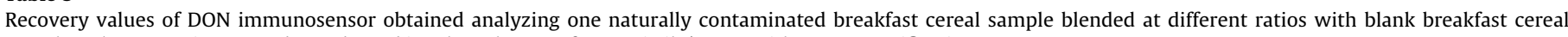
samples. The extraction procedure adopted involves the use of acetonitrile/water without any purification step.

\begin{tabular}{|c|c|c|c|c|c|}
\hline Extraction solvent & $\begin{array}{l}\text { Ratio of naturally contaminated } \\
\text { sample/blank sample }\end{array}$ & $\begin{array}{l}\text { Endogenous DON } \\
\text { content } \pm \mathrm{SD}(\mu \mathrm{g} / \mathrm{kg})\end{array}$ & $\begin{array}{l}\text { DON found } \pm \text { SD } \\
(\mu \mathrm{g} / \mathrm{kg})\end{array}$ & $\mathrm{RSD}_{\mathrm{r}} \%$ & Recovery \% \\
\hline \multirow[t]{3}{*}{ Acetonitrile/water (84/16) } & $4 / 0$ & $485 \pm 61$ & $475 \pm 80$ & 16 & 98 \\
\hline & $1 / 3$ & $364 \pm 47$ & $432 \pm 55$ & 13 & 119 \\
\hline & $2 / 2$ & $242 \pm 31$ & $201 \pm 17$ & 8 & 83 \\
\hline
\end{tabular}


Of note, the use of a novel multielectrode strip which is used only as interrogating surface after the immunological chain offers the concrete possibility of multianalytes detection. Moreover, the fact that electrode surfaces can be easily miniaturized in a cost-effective and mass producible way opens the future to the possible development of in situ detection system using a portable electrochemical instrument and miniaturized strip for a lab-on-a-chip approach. Despite the sensitivity and selectivity of the immunosensors developed in this paper are comparable to those achieved with classic ELISA approach or with SPR, the above cited advantages make the electrochemical platform one of the most valid alternative to such techniques. Future work will be targeted in this direction with the production of multi-array miniaturized printed sensors which will be challenged with multiple analytes.

\section{Acknowledgement}

The authors wish to thank the European Commission (New technologies to screen multiple chemical contaminants in foods: Contract FOOD-CT-2005-006988, project acronym BioCop) for financial support.

\section{Appendix A. Supplementary data}

Supplementary data associated with this article can be found, in the online version, at doi:10.1016/j.bios.2010.04.029.

\section{References}

Anklam, E., Stroka, J., Boenke, A., 2002. Food Control 13 (3), 173-183.

Bennett, R.W., 2008. J. Rapid Met. Autom. Microb. 16 (4), 320-329.

Bretz, M., Beyer, M., Cramer, B., Humpf, H.U., 2006. Mol. Nutr. Food Res. 50, 251-260.

Cahill, L.M., Kruger, S.C., McAlice, B.T., Ramsey, C.S., Prioli, R., Kohn, B., 1999. J. Chromatogr. A 859 (1), 23-28.

Commission Regulation N. 1126/2007 of 28 September 2007. Off. J. Eur. Union L 255/14.

D’Mello, J.P.F., Placinta, C.M., Macdonald, A.M.C., 1999. Anim. Feed Sci. Technol. 80 (3-4), 183-205.

Delibato, E., Bancone, M., Volpe, G., De Medici, D., Moscone, D., Palleschi, G., 2005. Anal. Lett. 38, 1569.
Dong, J., Ihara, M., Ueda, H., 2009. Anal. Biochem. 386 (1), 36-44.

Gehring, A.G., Brewster, J.D., Irwin, P.L., Tu, S.I., Van Houten, L.J., 1999. J. Electroanal. Chem. 469 (1), 27-33.

Haouet, N.M., Altissimi, S., 2003. Webzine Sanità Pubblica Veterinaria. 19, 4-5. http://www.biocop.org.

Helali, S., Martelet, C., Abdelghani, A., Maaref, M.A., Jaffrezic-Renault, N., 2006. Electrochim. Acta 51 (24), 5182-5186.

Klötzel, M., Schmidt, S., Lauber, U., Thielert, G., Humpf, H.U., 2005. Chromatographia $62(1-2), 41-48$.

Kolosova, A.Y., De Saeger, S., Sibanda, L., Verheijen, R., Van Peteghem, C., 2007. Anal. Bioanal. Chem. 389 (7-8), 2103-2107.

Korpimäki, T., Hagren, V., Brockmann, E.-C., Tuomola, M., 2004. Anal. Chem. 76 (11), 3091-3098.

Krska, R., Baumagartner, S., Josephs, R., 2001. Fresen. J. Anal. Chem. 371 (3), 285-299.

Lattanzio, V.M.T., Pascale, M., Visconti, A., 2009. TrAC Trends Anal. Chem. 28 (6), $758-768$.

Li, X., Yang, X., Zhang, S., 2008. TRAC Trends Anal. Chem. 27 (6), 543-553.

Maragos, C.M., McCormick, S.P., 2000. Food Agric. Immunol. 12, 181-192.

Maragos, C.M., Plattner, R.D., 2002. J. Agric. Food Chem. 50 (7), 1827-1832.

Milanez, T.V., Valente-Soares, L.M., 2006. J. Braz. Chem. Soc. 17 (2), 412-416.

Nassef, H.M., Civit, L., Fragoso, A., O'Sullivan, C.K., 2009. Anal. Chem. 81 (13), 5299-5307.

Nicol, M.J., Lauren, D.R., Miles, C.O., Jones, W.T., 1993. Food Agric. Immun. 5 (4), 199-209.

Näreoja, T., Vehniäinen, M., Lamminmäki, U., Hänninen, P.E., Härmä, H., 2009. J Immunol. Methods 345 (1-2), 80-89.

Ngundi, M.M., Qadri, S.A., Wallace, E.V., Moore, M.H., Lassman, M.E., Shriver-Lake, L.C., Ligler, F.S., Taitt, C.R., 2006. Environ. Sci. Technol. 40 (7), 2352-2356.

Olsson, J., Börjesson, T., Lundstedt, T., Schnürer, J., 2002. Int. J. Food Microb. 72 (3), 203-214.

Piermarini, S., Micheli, L., Ammida, N.H.S., Palleschi, G., Moscone, D., 2007. Biosens. Bioelectron. 22 (7), 1434-1440.

Piermarini, S., Volpe, G., Micheli, L., Moscone, D., Palleschi, G., 2009. Food Control 20 (4), 371-375.

Ricci, F., Amine, A., Palleschi, G., Moscone, D., 2003. Biosens. Bioelectron. 18 (2-3), $165-174$.

Ricci, F., Arduini, F., Tuta, C.S., Sozzo, U., Moscone, D., Amine, A., Palleschi, G., 2006. Anal. Chim. Acta 558 (1-2), 164-170.

Ricci, F., Volpe, G., Micheli, L., Palleschi, G., 2007. Anal. Chim. Acta 605 (2), 111-129.

Schneider, E., Curtui, V., Seidler, C., Dietrich, R., Usleber, E., Märtlbauer, E., 2004. Toxicol. Lett. $153(1), 113-121$.

Schnerr, H., Vogel, R.F., Niessen, L., 2002. Food Agric. Immunol. 14 (4), 313-321.

Sinha, R.C., Savard, M.E., 1996. Can. J. Plant Pathol. 18, 233-236.

Tüdös, A.J., Lucas-Van Den Bos, E.R., Stigter, E.C.A., 2003. J. Agric. Food Chem. 51 (20), 5843-5848.

Valjakka, J., Hemminki, A., Niemi, S., S“oderlund, H., Takkinen, K., Bouvinen, J., 2002. J. Biol. Chem. 277 (46), 44021-44027.

Vallina-García, R., del Mar García-Suaǐrez, M., Fernaǐndez-Abedul, M.T., Meřndez, F.J., Costa-García, A., 2007. Biosens. Bioelectron. 23 (2), 210-217. 